Itinéraires Itinéraires

Littérature, textes, cultures

\title{
La correspondance de Jacques Rivière et Alain- Fournier : une aventure de la lecture
}

Julie Aucagne

\section{OpenEdition}

\section{Journals}

Édition électronique

URL : https://journals.openedition.org/itineraires/2166

DOI : 10.4000/itineraires.2166

ISSN : 2427-920X

Éditeur

Pléiade

\section{Édition imprimée}

Date de publication : 1 mai 2010

Pagination : 71-86

ISBN : 978-2-296-11224-7

ISSN : 2100-1340

\section{Référence électronique}

Julie Aucagne, "La correspondance de Jacques Rivière et Alain-Fournier : une aventure de la lecture », Itinéraires [En ligne], 2010-1 | 2010, mis en ligne le 01 mai 2010, consulté le 28 juin 2022. URL : http:// journals.openedition.org/itineraires/2166 ; DOI : https://doi.org/10.4000/itineraires.2166

\section{cc)}

Itinéraires est mis à disposition selon les termes de la licence Creative Commons Attribution - Pas d'Utilisation Commerciale - Pas de Modification 4.0 International. 


\title{
La correspondance de Jacques Rivière et Alain-Fournier : une aventure de la lecture
}

\begin{abstract}
Through a precise study of the letters that Jacques Rivière and Alain-Fournier wrote to each other between 1904 and 1909, when they were young men, the article uncovers a form of existential event in the very act of reading. It analyzes the progressive separation between two literary and spiritual personalities around a practice they first shared. It inscribes reading in a configuration of vocation, and in the form of destiny : living, reading, being read, are but different aspects of one single tension towards the future.
\end{abstract}

Keywords : Jacques Rivière, Alain-Fournier, correspondence, vocation, reading Mots clés : Jacques Rivière, Alain-Fournier, correspondance, vocation, lecture

Jacques Rivière et Henri Fournier ${ }^{1}$ se sont connus en octobre 1903 au lycée Lakanal de Sceaux, où ils étaient entrés pour préparer le concours d'entrée à l'École normale. La rencontre n'eut apparemment rien d'un coup de foudre : dans l'essai précédent l'édition posthume des Miracles d'Alain-Fournier ${ }^{2}$, Jacques Rivière explique qu'en dépit d'une vague attirance mutuelle, ils avaient plutôt tendance à s'agacer de leurs différences : lui, timide et « appliqué », se scandalisait un peu du tempérament « libre et joueur » et de l'esprit de révolte de Fournier, tandis que celui-ci le provoquait à plaisir ${ }^{3}$. En guise de point de départ, il est bon de faire un détour par un passage capital de cet essai écrit à la lumière de la Correspondance ${ }^{4}$.

1. Nous utilisons l'édition de la Correspondance établie par Alain Rivière et Pierre de Gaulmyn, parue chez Gallimard en 1991, en deux volumes. Alain-Fournier y étant généralement désigné par son véritable nom, Henri Fournier, aussi bien dans les lettres que dans l'appareil critique, nous conserverons ce parti-pris, sauf si le contexte le justifie.

2. Alain-Fournier, par Jacques Rivière [1924], dans Alain-Fournier, Miracles, Paris, Fayard, 1986, p. 11-81.

3. Voir ibid., p. 13-14.

4. Voir ibid., p. 30 où Rivière parle d'« une énorme correspondance, qui [lui] permet aujourd'hui 

lecture :

Il montre en effet qu'entre les deux hommes, tout commença par une

Je date des environs de Noël 1903 la révélation qui nous fut faite en même temps l'un à l'autre. Pour nous remercier du compliment traditionnel que nous lui avions adressé avant le départ en vacances, notre excellent professeur, M. Francisque Vial, [...] nous fit une lecture du Tel qu'en songe d'Henri de Régnier :

J'ai cru voir ma Tristesse - dit-il - et je l'ai vue

- Dit-il plus bas -

Elle était nue,

Assise dans la grotte la plus silencieuse

De mes plus intérieures pensées, ....etc.

$[\ldots]$

Nous tombions, sans avoir même su qu'il en existât de tels, sur des mots choisis exprès pour nous et qui non seulement caressaient nommément notre sensibilité, mais encore nous révélaient à nous-mêmes. Quelque chose d'inconnu, en effet, était atteint dans nos âmes; une harpe que nous ne soupçonnions pas en nous s'éveillait, répondait; ses vibrations nous emplissaient. Nous n'écoutions plus le sens des phrases; nous retentissions seulement, devenus tout entiers harmoniques. Je regardais Fournier sur son banc; il écoutait profondément; plusieurs fois nous échangeâmes des regards brillants d'émotion. À la fin de la classe, nous nous précipitâmes l'un vers l'autre. Les forts en thème ricanaient autour de nous, parlaient avec dédain de « loufoqueries ». Mais nous, nous étions dans l'enchantement et bouleversés d'un enthousiasme si pareil que notre amitié en fut brusquement portée à son comble ${ }^{5}$.

Le récit de Rivière confère à cet épisode le statut d'une épiphanie : on observera d'abord qu'ici la lecture parvient aux deux amis, inscrivant inévitablement au sein de l'essai biographique la figure de la vocation; mais le récit de l'événement esquisse aussi un certain nombre de traits de définition de la lecture - ou plutôt il cerne d'ores et déjà quelques aspects de ses pratiques possibles et de sa fonction au sein de la vie des jeunes gens.

D'abord, la lecture s'impose comme lien : si lire, c'est établir des relations - entre deux systèmes de signes notamment - il est évident ici que cette définition s'étend du domaine purement cognitif au domaine existentiel en liant entre eux Rivière et Fournier, d'un lien indéfectible et presque magiquement noué. Elle crée en outre, en même temps qu'une amitié, une communauté, par le sentiment de reconnaissance qu'elle suscite à plusieurs niveaux : entre l'auditoire et le poète, et entre les membres de l'auditoire eux-mêmes dont s'excluent naturellement, par leurs ricanements, les béotiens, les « forts en thème ». Comme révélation et comme initiation, elle s'inscrit directement 
dans le récit rétrospectif comme ce que Pierre de Gaulmyn appelle, parlant des « événements ordinaires » racontés au fil de la correspondance, une « [figure] de l'apprentissage de la vie et de l'œuvre $^{6} \gg$; pour Fournier et Rivière, la lecture ne sera jamais une activité purement désincarnée ni totalement solitaire : elle fera partie intégrante de la trajectoire existentielle, qu'elle détermine comme une trajectoire double, qui s'accomplit dans la relation à l'autre en parlant des « événements ordinaires » racontés au fil de la correspondance :

En tant que « figure de l'apprentissage », la lecture correspond ici à ce que l'on pourrait appeler l'entrée dans le monde : les « textes admirables » que les lycéens connaissaient jusque-là étaient entourés, écrit Rivière, d'une « gangue scolaire [qui] emprisonnait aussi leur sortilège ». Il ajoute :

Et puis ni Racine, ni Rousseau, ni Chateaubriand, ni même Flaubert ne s'adressaient à nous, jeunes gens de 1903 ; ils parlaient à l'humanité universelle; ils n'avaient pas cette voix comme à l'avance dirigée vers notre cœur, que tout à coup Henri de Régnier nous fit entendre ${ }^{7}$.

Ce que décrit l'épisode, c'est donc aussi tout simplement la découverte de l'existence d'une littérature contemporaine : l'activité de lecture sort alors des sentiers battus de la tradition, de ce que Sartre appelle la « littérature faite », et cesse d'être guidée par le poids d'une histoire déjà accomplie et comprise, et d'une série limitée de grands noms. Ce que liront Rivière et Fournier, c'est avant tout la littérature de leur temps, ce qui fait dire encore à Pierre de Gaulmyn que la correspondance donne à voir « le parcours de lecture d'une époque ${ }^{8} \gg-$ non seulement dans ce qui est lu mais, nous le verrons, dans la manière de le lire. Ce qui en effet marque la plupart des grandes découvertes relatées dans la correspondance, c'est la joie d'être le premier à lire, de faire partie des découvreurs de Barrès, Laforgue ou Claudel, de jouer à son tour les initiateurs après s'être jeté dans le monde vivant, grouillant, et « encore muet » de la littérature à faire, qui n'attend pour se réaliser qu'un lecteur disponible, prêt à s'immerger dans un univers encore vierge de signes mystérieux parce que sans histoire et, faute de lecteur, inaccomplis. C'est en ce sens que la lecture est également une figure de l'aventure : les deux lecteurs de la Correspondance apparaissent face aux livres comme les acteurs du roman - auteur, lecteur et personnage - que décrit Rivière : en état de perpétuelle disponibilité à la découverte.

Ce que l'épisode fondateur met également en évidence, et que la correspondance confirme sans cesse, c'est que la lecture est rencontre : elle survient au moment où on ne l'attend pas, et elle influe sur le cours de l'existence parce qu'elle est confrontation à un autre dont on ne sait pas s'il sera frère, complice ou adversaire, auquel on n'a d'autre choix que de faire face pour finalement

6. Pierre de Gaulmyn, préface à la Correspondance, op. cit., t. I, p. 9-21 et p. 16.

7. Jacques Rivière, Alain-Fournier, op. cit., p. 17.

8. Pierre de Gaulmyn, préface à la Correspondance, op. cit., t. I, p. 21. 
se l'incorporer, le dépasser ou le battre. La correspondance peut ainsi se lire comme l'histoire de ces rencontres, qui dessinent un parcours chaotique, qui s'apparente souvent à une errance et qui parfois débouche, « au bout d'une longue avenue sombre, sur un rond de lumière tout petit ${ }^{9} »$; elle donne à voir un ensemble de pratiques qui sont autant de façon de composer avec l'altérité, avec l'inconnu et qui, en s'écrivant, cherchent aussi à se ressaisir de l'expérience du lecteur-épistolier comme itinéraire, et de sa relation à l'autre comme caisse de résonance et facteur déterminant de son évolution.

Car ce dont la correspondance garde la trace enfin, c'est d'une double expérience de lecture, ou plutôt d'une expérience exponentielle; bien sûr, à deux épistoliers, deux manières et deux itinéraires; mais ce qui doit nous retenir surtout c'est qu'ici, à tout moment, le lecteur s'expose à son tour à être lu : sous le regard de l'autre, l'activité intime et silencieuse qu'est traditionnellement la lecture se métamorphose en pratique sociale, devient parade, drame; finalement l'on se regarde lire et l'on s'écrit comme lecteur, transformant chaque compte-rendu en épisode de l'aventure existentielle - simple anecdote, ou véritable tournant. Sous le regard de l'autre, lecteur souvent plus interloqué que complice, on parcourt la somme de ses vies possibles, n'évitant ni les errements, ni les fausses pistes, au milieu des éblouissements. C'est en ce sens aussi que la Correspondance rappelle le Roman d'aventure idéal de Rivière : parce qu'elle inclut nécessairement, dans son fonctionnement, l'existence d'un lecteur, qu'elle s'écrit à la fois pour lui et en dépit de lui; à chaque lettre, elle le prend et l'immerge dans un univers qui lui résiste et le livre aux tourments et aux délices de l'altérité et de l'inaccompli, à l' " émotion » d'être « sur le bord de ce qui n'existe pas encore ${ }^{10} »$.

Cette expérience de l'inconnu comme composante essentielle de la lecture n'est cependant pas première, comme on l'a vu à travers le récit de Rivière : au contraire, dans les premiers mois de la Correspondance, la lecture est encore souvent recherche de reconnaissance et de communion; elle ne se conçoit encore que sur le modèle de l'expérience fondatrice, comme dans cette lettre du 25 août 1904 où Fournier, après avoir copié quelques vers de Laforgue, écrit à son ami : " Admire, va - admire, ce sera "sympathiser" avec moi ${ }^{11}$. » Et Rivière, parlant de Maeterlinck: « Si tu connais Sagesse et destinée, tu dois sentir combien j'en ai été pénétré; mais on n'est jamais pénétré d'un livre, que si on l'avait obscurément pensé avant de le lire $^{12}$. \ Ces réflexions montrent que la fonction initiale de la lecture est de fabriquer de l'identité, dans tous les sens du terme : en 1924, Rivière évoque le symbolisme comme une sorte de cocon, « un lieu ravissant d'exil, ou de

9. L'expression est d'Alain-Fournier dans Le Grand Meaulnes [1913], Paris, Fayard, 1997, p. 120.

10. Jacques Rivière, Le Roman d'aventure [1913], Paris, Éditions des Syrtes, 2000, p. 74.

11. Correspondance I, Henri Fournier à Jacques Rivière, 22 janvier 1905, p. 53.

12. Correspondance I, Jacques Rivière à Henri Fournier, juillet 1904, p. 31-32. 
rapatriement plutôt, un paradis ${ }^{13} »$, au . sein duquel la lecture est encore communication pure, ciment et garant d'une harmonie générale. En cela, elle est forcément une expérience immédiatement partageable : dans les premières lettres, il suffit que figurent quelques bribes de vers - que l'autre complètera sans peine - pour qu'immédiatement se reforme le lien menacé par la séparation physique. Si pour les non-initiés ces vers isolés, détachés de leur contexte, sont passablement hermétiques, pour les correspondants ce sont des signes de reconnaissance, les symboles transparents d'une communauté d'âme qui les oppose au reste du monde, les constitue comme élite, la citation revêtant alors une fonction incantatoire. La lecture symboliste, telle que Rivière la décrira dans Le Roman d'aventure, est alors encore un modèle opérant : « les émotions qu'ils [l'auteur symboliste et son lecteur] ont eu séparément - et l'un plus clairement que l'autre - en face de tel ou tel spectacle bien connu de tous deux, voici qu'ils les revivent ensemble ${ }^{14}$. » Pendant les quelques vacances de l'année 1904-1905, cela est vrai aussi des lecteurs complices que sont encore Jacques Rivière et Henri Fournier.

Cependant ce modèle n'est durable ni, si l'on en croit Rivière, dans l'histoire littéraire, ni à l'échelle des vies particulières. D'abord, ce qui fonde le « pacte épistolaire ${ }^{15}$ » des deux amis, c'est précisément la longue période de séparation qui commence pour eux en juillet 1905 : or il s'avère bientôt que la correspondance fidèlement entretenue est impuissante à maintenir bien longtemps l'intégrité du « paradis » où ils baignaient ensemble; un mois seulement après que Rivière a quitté Lakanal, Fournier lui écrit :

Le seul petit chagrin que m'a fait ta critique ç'a été de voir que je m'étais un peu trompé lorsque je me disais : « on se relit ces vers, on les admire tous deux, on ne se dit rien, mais on comprend pourquoi on les admire - c'est si évident et nous nous comprenons si bien. » Déception que je m'efforce de rattraper, de réparer maintenant ${ }^{16}$.

Or réparer désormais, c'est expliquer, démontrer, plaider plutôt qu'évoquer; la relation à l'autre a perdu de sa parfaite symétrie, de son immédiateté : la distance physique impose aussi une prise de distance intellectuelle avec ce qui est lu; pourtant, l'on ne perd pas forcément au change, comme Jacques Rivière le concède :

13. Jacques Rivière, Alain-Fournier, op. cit., p. 18.

14. Jacques Rivière, Le Roman d'aventure, op. cit., p. 19.

15. «Selon ce pacte, ils s'enverraient [...] réciproquement la liste de leurs lectures, des tableaux vus et des concerts entendus, avec les commentaires qui leur viendraient à l'esprit. » Préface à la Correspondance, op.cit., p. 10.

16. Correspondance I, Henri Fournier à Jacques Rivière, 27 août 1905, p. 111. La critique de Rivière visait les auteurs favoris de Fournier, Laforgue et Jammes. 
Nous aurions causé là-dessus. Écrit, c'est mort. Il est vrai que je rapporte d'autres choses que j'écris mais que je n'aurais pas pu dire ${ }^{17}$.

Si l'apprenti critique ne rompt pas sans plaisir avec l'âge d'or, c'est entre autres parce qu'il sait qu'il lui reste un monde à découvrir : les années 1906-1907 représentent ainsi pour les deux épistoliers une période de grande fécondité, qui se nourrit de l'exacerbation des divergences de goût entre les deux jeunes gens : car Rivière se passionne soudain pour Barrès, tandis que Fournier reste globalement fidèle à ses anciennes amours. Mais ces divergences n'expliquent pas seules la différenciation progressive et l'enrichissement des pratiques de lecture; c'est plutôt l'inverse qui se produit, comme si les livres préférés étaient élus en fonction de l'usage que l'on compte en faire. Les itinéraires séparés de l'un et de l'autre dessinent ainsi, par leurs entrecroisements et leurs écarts, un répertoire des manières de lire dont l'élargissement, les errements, les trouvailles, sont pour tous deux le préliminaire obligé de l'invention d'un langage propre qui les projette, acteurs cette fois, dans le champ grand ouvert de la littérature contemporaine.

Le débat sur Barrès, qui les occupe six mois durant, de septembre 1905 au printemps 1906, est à cet égard particulièrement riche d'enseignement pour chacun. Du côté de Rivière la découverte est aussi stupéfiante, au sens propre du terme, que délicieuse :
Barrès.
Il m'est très difficile de t'en parler, tant je le comprends. [... ] Je ne m'arrêterai pas avant d'avoir tout lu. C'est évidemment avec Samson et Dalila, Debussy et Maeterlinck une des grandes révélations de ma vie. [...] Et tiens! je vais en rester là. Je ne peux vraiment rien dire sur lui. Faismoi toutes tes objections, dis-moi quelles croyances « philosophiques et littéraires » mon admiration pour lui choque en toi. Alors je répondrai. Mais pour le moment j'ai honte et je souffre d'avoir parlé si lourdement de Celui qui est aussi subtil et profond que l'essence du feu ${ }^{18}$.

Six mois plus tard, l'expérience se renouvelle presque à l'identique avec Claudel :

Maintenant je devrais te parler de Claudel, dont j'étouffe, bien que je ne l'aie presque pas relu depuis ma dernière lettre. Il fait son œuvre en moi et sourdement transforme tous mes gestes, ou plutôt la signification que j'attribue à tous mes gestes. Mais je ne sais pas si je suis assez bien disposé aujourd'hui pour en parler décemment. Je vais sortir. Au retour je verrai.

Oui. Mais par où commencer? Par où aborder l'effroyable génie ${ }^{19}$ ?

17. Correspondance I, Jacques Rivière à Henri Fournier, 24 juin 1906, p. 434.

18. Correspondance I, Jacques Rivière à Henri Fournier, 20 septembre (« et jours suivants ») 1905, p. 150.

19. Correspondance I, Jacques Rivière à Henri Fournier, 5 avril 1906, p. 347-348. 
Les données de départ sont toujours les mêmes : mais désormais à l'éblouissement, à la « révélation » succède un silence embarrassé. C'est qu'il ne suffit plus de répéter les mots de l'auteur pour que l'autre lecteur communie avec vous dans l'admiration : la lecture s'est repliée sur le champ de l'intime, et refuse apparemment d'en sortir. Désormais l'expérience vous laisse seul, bouleversé et muet, avec votre toute nouvelle étrangeté :

J'ai relu hier La Porte étroite; j'en étais malade. Sans doute je comprends bien que cela me concerne spécialement, que je n'ai pas le droit d'exiger des autres le même enthousiasme. Mais pourtant je voudrais le trouver en eux; j'aimerais tant que ceux que j'aime, subissent cela comme moi ${ }^{20}$.

Revenant vers son ami presque méconnaissable, Rivière éprouve à travers la correspondance une première expérience de l'incommunicable. Aussi, très vite, les lettres à l'autre autrefois complice et désormais lointain deviennent le lieu où doit s'inventer la critique, c'est-à-dire un langage de médiation. Il lui faut désormais, pour tenter d'annuler l'espace qui le sépare de Fournier, et au milieu duquel se dresse comme un bloc infranchissable l'hermétisme du livre aimé, déplier entre eux, devant eux, le réseau énigmatique et serré de l'œuvre, pour la faire enfin com-prendre.

Jacques Rivière va donc se poser comme celui qui, des deux, est le « métaphysicien », le philosophe; expliquer, exposer pour l'autre les idées que sont supposées contenir les œuvres littéraires, voilà la tâche qu'il s'est assignée. Or dans ce domaine Fournier est un interlocuteur exigeant, qui n'a de cesse de pousser son ami dans ses derniers retranchements - d'abord parce que l'œuvre analysable lui est automatiquement suspecte; Rivière notera dans l'essai qu'il lui consacre :

Fournier aperçoit un inconvénient grave pour lui dans toute opération de discernement ou même d'abstraction; elle isole, elle brise un contact, pense-t-il ${ }^{21}$.

Pour Rivière - dans un premier temps tout au moins - la possibilité d'analyser l'œuvre est au contraire le signe de sa profondeur, la preuve de son pouvoir d'ordonnancement et d'éclaircissement du moi et du monde :

Je touche ici à ce que j'ai appris de plus précieux de Barrès, au grand service qu'il m'a rendu. [...] [Il] m'a appris à « départager ce qui choque mes seuls préjugés et ce qui offense réellement mes délicatesses ». Il m’a appris à m'occuper plus soigneusement de ma tenue morale, à me « composer » davantage. Bienfait inestimable, et dont je sens déjà l'infinie portée ${ }^{22}$.

20. Correspondance II, Jacques Rivière à Henri Fournier, 8 avril 1909, p. 269.

21. Alain-Fournier, op.cit., p. 33.

22. Correspondance I, Jacques Rivière à Henri Fournier, 20 septembre (« et jours suivants ») 1905, p. 164. 
Fournier ne manquera pas de reconnaître dans ce discours, non seulement un acte d'allégeance peut-être néfaste ${ }^{23}$, mais aussi un renoncement à son intégrité puisqu'il s'agit de « départager », de « se composer »; aussi reste-t-il sur la défensive :

Tu n'ose pas dire encore que j'aime Barrès. Il me semble qu'il est trop tard maintenant et qu'au moment où j'aurais pu l'aimer et le suivre, il n'est pas venu $[\ldots .$. . Je trouverai toujours que toutes ces opinions [i.e. philosophiques et littéraires] même énoncées le plus brillamment du monde, ne valent pas seulement un vers de moi ou d'un garçon sincère quelconque. [...] «Il reste que» $[\ldots]$ tes lettres sont des merveilles de lucidité, d'intelligence. (J'y prends autant de plaisir qu'aux vers du garçon le plus sincère) et je crois [...] que Barrès n'a pas encore trouvé de critique aussi lumineux. Il t'attend. Dépêche-toi ${ }^{24}$.

L'éloge de « l'intelligence » de Rivière est ici redoutable : Fournier n'avait-il pas affirmé quelques jours plus tôt et non sans volonté de provocation : «j'[ai] pour l'intelligence, ou ce qu'on appelle comme ça, le plus profond mépris ${ }^{25} » ; l^{\prime}$ « intelligence », ce n'est peut-être que la démonstration d'une incapacité à « sentir » la vie réelle :

Je n'aime pas qu'on crée et habille des abstractions, qu'on fasse se promener la Sensibilité au bras de l'Amour [...] : objection $n^{\circ} 1$ aux Barbares de Barrès. Je trouve tout cela d'un froid, d'un faux! Qu'est-ce que c'est que ça la Femme? l'Amour? [...] ce système ne vaut que pour Barrès, il est inattaquable puisqu'idéaliste, évidemment, mais de quel droit arrêter, symboliser, arrêter la vie ondoyante et diverse ${ }^{26}$ ?

À quoi Rivière réplique : « ne faut-il pas - avoue-le - qu'il y ait quelque chose de sérieux dans Barrès puisque depuis deux mois nous nous escrimons sur ses œuvres et ne pouvons nous les ôter de la mémoire. » Il ajoute :

Il y a dans notre dispute - je crois - un malentendu général. Tu juges Barrès au point de vue purement littéraire, moi au point de vue philosophique, tu le regardes comme un simple écrivain, moi comme un maître; tu apprécies son talent, moi sa pensée. [...] aujourd'hui tu dis : « Je n'aime pas qu'on crée et qu'on habille des abstractions ». Très bien, mais ça veut dire que tu n'aimes

23. Voir par exemple la lettre du 22 janvier 1906, Correspondance I, p. 248-249 : « Tu m'agaces, quand tu prends dans Barrès ce qui très certainement ne peut pas être toi. Mais je ne sais pas si je peux te le dire... de temps en temps, quand tu te dis avec délices : je vais dire cela parce que Barrès l'aurait dit, tu m'agaces. »

24. Correspondance I, Henri Fournier à Jacques Rivière, 4 octobre 1905, p. 169.

25. Correspondance I, Henri Fournier à Jacques Rivière, 23 septembre 1905, p. 155.

26. Correspondance I, Henri Fournier à Jacques Rivière, 2 novembre 1905, p. 195. 
pas la mise en scène de sa pensée, de ses analyses; mais il faut que tu juges cette pensée, en-dehors des formes, qui te déplaisent ${ }^{27}$.

Juger en-dehors des formes : une aberration, selon Fournier, pour qui la littérature devrait être le contraire de la concrétisation en images de rêves et de désirs que décrit Rivière dans la même lettre - une réalité indiscutable, aussi tangible que la vie même; il n'existe pas de monde idéal, de " pensée " pure qui préexisterait à sa mise en mots et en images. La vérité de l'œuvre littéraire n'est pas au-delà de la surface fascinante, sidérante, du texte lu, comme une réalité profonde dont il ne serait que l'indice ou le vêtement chatoyant, séduisant : la vérité, c'est ce texte même. Aux yeux de Fournier d'ailleurs, Rivière ne s'y trompe pas totalement; ce que Barrès flatte en lui, c'est son goût de la spéculation, presque de la dissection, et il s'y complaît momentanément sachant qu'il dépassera bientôt le système inventé pour le lire. Aussi, quand Rivière propose un peu plus tard d'envoyer à Fournier ses notes sur Claudel, il s'en excuse presque :

Elles t'agaceront parce qu'elles sont écrites dans un style froid, mort, décoloré. Mais j'ai voulu simplement analyser, décomposer, distinguer les durs moments de la pensée avant de la recomposer, de lui redonner vie par la synthèse. C'était un travail difficile et dangereux, mais indispensable et bien passionnant. Maintenant je manque du courage et du temps nécessaire pour reconstruire Claudel. Et si je ne le fais pas à présent, je ne le ferai jamais ${ }^{28}$.

Fournier réplique :

Qu'est-ce que tes notes sinon la joie inquiète de faire entrer tout dans le même moule, de condenser toutes les vérités dans la même, puis, en conclusion, de trouver fausse l'unique et ultime vérité; ou encore de trouver identiques le préambule et la conclusion, ou encore de les trouver à la fois logiquement déduites et rigoureusement contradictoires. Puis, par moments, l'inquiétude de sentir que tout cela craque et qu'il y a autre chose ${ }^{29}$.

Non que Fournier désapprouve la critique par elle-même : ce qui l'agace c'est cette « manie scolaire ${ }^{30}$ » toujours sensible, selon lui, dans la manière dont Rivière pratique la glose, toujours en dessous de ce que lui apporte l'expérience réelle de la lecture faisant partie de l'expérience vitale : il y a trop loin de la révélation qu'il ressent au moment de la première lecture au langage qu'il utilise pour en rendre compte. Sur le chemin où il s'avance, la critique ne consiste qu'à dépouiller les idées de leurs oripeaux littéraires pour les ramener au grand jour, dénudées : et cet effort de l'intelligence les tue. Comment, alors, parler de ce qui vous hante, dont on ne parvient pas

27. Correspondance I, Jacques Rivière à Henri Fournier, 5 novembre 1905, p. 198-199.

28. Correspondance I, Jacques Rivière à Henri Fournier, 27 mai 1906, p. 419-420.

29. Correspondance I, Henri Fournier à Jacques Rivière, 19 novembre 1906, p. 577.

30. Ibid. 
à se désolidariser, et qui en outre reste insaisissable de par son caractère « ondoyant et divers », comme l'est la vie même? Le premier modèle de lecture de Rivière est donc une fausse piste, une initiation mais non un aboutissement, dont les limites lui apparaissent bientôt, comme Fournier le lui avait prédit; mais c'est Claudel qui se chargera de la démonstration :

De tout ce que je t'ai écrit sur Claudel, je ne considère rien comme définitif. Ma première lettre sur lui (primitivité moderne, etc.) je n'en garde rien. Tout cela tournait autour, ne pénétrait pas. [...] Entre autres je ne peux plus dire qu'il ait voulu dramatiser des idées, qu'il ait cherché à exprimer poétiquement des idées philosophiques. Cela est atrocement faux. Non. Il se trouve que son œuvre manifeste des idées, qui sont des sentiments. [...] Claudel grandit en moi. Ce n'ai pas moi qui l'ai pénétré. Si j'ai paru m'enthousiasmer sur ses « idées », c'est que j'ai dû pauvrement traduire en langage idéologique, ce qu'il manifestait profondément par la puissance de son art. Au fond je suis imprégné par lui d'un enseignement sans forme et sans mots, qui commande toute ma vie intérieure ${ }^{31}$.

Cette propension à se laisser habiter par les œuvres des autres n'est pas une découverte : la période « Barrès » la lui avait déjà révélée, sur un mode plus souriant mais déjà inquiet :

Je ris de moi quand je considère mon effroyable plasticité. Dès que je trouve une pensée, qui ressemble à la mienne, je m'abandonne à elle. Je prends tous les contours qu'elle m'impose. Je suis délicieusement inerte. Et quand je me regarde, je me demande pourtant avec effroi si j'ai quelque chose d'original, tant je deviens avec facilité. Je crois que ma pensée passera par toutes les formes que peut prendre une pensée. Mais s'arrêtera-telle jamais ${ }^{32}$ ?

Cette « plasticité » est une sorte de plaisir coupable : une compréhension intime, fusionnelle des œuvres qui le fait s'y abandonner avec volupté - mais une compréhension muette, inutile et dissolvante. En outre, puisque chaque lecture est une perte de soi - en admettant que ce « moi » soit autre chose que les formes successives qu'il adopte au hasard des rencontres - l'analyse, ou deuxième lecture, ne peut être qu'une tentative de rassemblement de soi à travers une discipline, une méthode. Il s'agit de « reconstruire », de réinsérer une armature rigide, une forme pure et lisible, à l'intérieur d'un ensemble mou, fluide, sans contours : une vérité, une et éternelle, une signification ou des significations formulables. Mais c'est un dilemme sans fin pour Rivière, pris entre deux tentations contradictoires : la compréhension parfaite mais indicible, ou l'explication partielle et fausse, mais exprimable. C'est alors qu'il ressent douloureusement sa différence avec Fournier : 
Tu souris un peu de me voir subir ainsi jusque dans ma vie et dans ma chair les influences. Toi, de tous ceux que tu admires, vite tu extrais ce qui t'est essentiel, ce qu'ils t'apportent de nouveau; car tu as ton œuvre à faire, et qui t'occupe trop pour te laisser voir ailleurs dans le monde autre chose que des matériaux. Mais moi, mais moi qui n'ai rien à faire que de comprendre, moi qui m'offre vide à toute invasion. Il faut que je subisse, que je me fasse d'abord la chair et l'âme de celui que j'accueille, afin de le comprendre, afin de le posséder. Et ensuite il faut que je me débarrasse de lui, pour accueillir encore ${ }^{33}$.

L'image finale montre combien il est difficile à Rivière de lutter contre la première de ces tendances : au temps délicieux de la hantise doit succéder un arrachement, presque un accouchement, qu'il redoute. Plusieurs mois après avoir constaté son impuissance à parler de Claudel, il n'en est toujours pas « débarrassé » : il croit alors devoir choisir entre le langage clair et insuffisant de celui qui explique, et les impressions muettes et profondes par lesquelles la lecture le modifie :

[...] comme tu dis, ou bien il faut être poète, et se dissoudre en toutes choses, et s'éparpiller sans mémoire, ou bien il faut être Claudel et « sentir l'ordre de la confusion » (admirablement juste ceci) - Or je ne suis ni poète ni Claudel. Donc...

Donc il ne me reste que ma petite tâche d'arrangeur, d'ajusteur, de constructeur logique. [...] Jadis tu disais : «Tu pourras être ce qu'est Mauclair»; oui, c'est cela. Le Monsieur qui met son esprit moyen, limpide et charmant au service des grands et des vrais, le Monsieur qui « expose » Edgar Pœ, Stéphane Mallarmé, Rodenbach et Paul Adam. Moi ce serait Claudel que j'exposerais. Et voilà tout.

Et pourtant, et pourtant, il faudra bien que mon désir s'écrie.

$[\ldots]$

Sentir l'ordre de la confusion. Serai-je assez grand ${ }^{34}$ ?

La résignation de Rivière à voir l'avènement du critique se payer de la mort de l'artiste, on le voit, n'est pas si évidente; comment renoncer à être celui qui sent et crée pour devenir celui qui pense et « expose »? Comment renoncer à soi, comment s'exclure de sa propre lecture?

Ce qui t'a blessé dans ma dernière lettre, je le sais très bien. C'est cette manière de considérer les idées et par suite leur expression, l'art comme quelque chose d'indépendant de moi-même, d'indifférent en soi, de vivant à part. $[\ldots]$

33. Correspondance II, Jacques Rivière à Henri Fournier, 5 juillet 1907, p. 56.

34. Correspondance I, Jacques Rivière à Henri Fournier, 29 novembre 1906, p. 585. Les citations font référence à la lettre d'Henri Fournier du 19 novembre, p. 578-579. 
Je sais bien que tu penses toujours à : « Nous ne séparerons pas la vie d'avec l'art. »

Mais moi je pense que c'est là une conception littéraire [...]. J'ai pu aimer ça. Mais je considère celui qui a écrit ça comme un comédien dont on s'amuse et qu'on méprise. Pour moi, si par hasard j'avais écrit quelque chose d'aussi renseignant sur ma vie, je ferais courir partout le bruit de ma mort et je changerais de nom ${ }^{35}$.

Cette palinodie apparente peut aussi s'interpréter comme rébellion contre l'invasion - consentie au départ - de sa personnalité par les livres, et montre bien quelle pression parfois insupportable Fournier l'artiste, l'intuitif, fait subir à son ami. Sa défiance envers toute médiation, défiance dont les lectures de Rivière paraissent confirmer sans cesse le bien-fondé, enferme parfois le critique dans d'insupportables apories. Mais comme il en est par ailleurs parfaitement conscient, cette tension vient de l'usage diamétralement différent que Fournier fait de ses lectures.

Comme on l'a rappelé, tandis que Rivière se précipite avec enthousiasme vers de nouveaux horizons, Fournier s'entête dans ses anciens goûts; on le voit copier, dans ses lettres, des passages entiers de Laforgue ou Jammes; on aurait tort pourtant de l'imaginer, sûr de lui et l'esprit statique, s'abîmant, en disciple soumis, dans une extase qui prolongerait sans fin le tout premier éblouissement. En réalité il conquiert, beaucoup plus rapidement que Rivière, une indépendance croissante; elle apparaît d'abord dans sa conception même du compte-rendu de lecture. Lorsqu'en réponse à une lettre remplie de citations Rivière le raille : «Je suis sûr que tu as créé Laforgue de toutes pièces. Tu me répondras que moi j'ai créé Barrès et il y aura du vrai $^{36} \gg-$ Fournier répond :

Ton reproche : "avoir créé Laforgue » est vrai en ce sens que je suis obligé de prendre de ci, de là, et seulement de loin en loin des bribes de vers pour le faire goûter à des profanes comme toi. - C'est-à-dire, plutôt, que je le recrée à votre usage ${ }^{37}$.

Cette pratique recèle chez Fournier deux postulations qui paraissent contradictoires et qui ne peuvent être en réalité que simultanées : la désinvolture par rapport à la lettre de l'œuvre, qui le fait la décomposer à loisir, est précisément ce qui le préserve de « subir » ses lectures; il semble ainsi pouvoir concrétiser, en lisant, ce paradoxe : faire de l'œuvre sa chose sans compromettre son intégrité. Ainsi son refus constant de l'analyse, par principe, presque par hygiène, fait-il de lui un lecteur-vampire - Rivière dira qu'il « pompait tous les sucs dont il avait besoin ${ }^{38} »-$ qui s'assimile ses

35. Correspondance II, 24 mai 1908, p. 182-184.

36. Correspondance I, Jacques Rivière à Henri Fournier, 13 janvier 1906, p. 245.

37. Correspondance I, Henri Fournier à Jacques Rivière, 22 janvier 1906, p. 257.

38. Alain-Fournier, op. cit., p. 30. 
lectures, se les incorpore, puis très rapidement les déborde et progresse ainsi, péniblement mais avec opiniâtreté, vers son identité et vers « [sa] forme ${ }^{39}$ ». La démarche n'a rien de narcissique : s'il se cherche dans les livres, ce n'est pas pour s'enchanter de sa découverte, mais pour aussitôt se dépasser. Le « moi » rencontré parfois au hasard des lectures se met à faire partie de son expérience, de la somme de ses possibles : il est le creuset où s'entassent expérience sur expérience, et d'où sortira l'œuvre unique.

Le reste est littérature :

Tu sais peut-être qu'un de mes idéals serait - s'il est formulable - d'aller droit au cœur de quiconque sans éveiller aucune idée de littérature (c'est-àdire de formulé, de pas senti ou d'au-dessus de la vie) ${ }^{40}$.

Si désormais Fournier assimile la littérature à l'artifice et qu'elle l'encombre, c'est qu'il vient de franchir une étape décisive vers sa vocation : il a toujours rêvé que dans le processus de lecture s'efface le média, que « rebondisse » en quelque sorte, dans l'âme d'autrui, sa propre expérience; il se met donc à lire comme il voudrait, lui, être lu; mais alors le simple éblouissement de la reconnaissance ne lui suffit plus, puisqu'il ne lit plus que pour écrire. Sa quête est autant prospective que réflexive : il veut que survienne ce qui, en lui, n'est présent que comme une virtualité encore secrète. C'est pourquoi il ne cherche pas de modèle - tout juste souhaite-til parfois trouver un guide qui le mènerait à la rencontre en lui de cet être potentiel et qui n'est pas encore advenu : Alain-Fournier, l'auteur. Claudel jouera un moment ce rôle, comme créateur d'un monde, c'est-à-dire comme anti-littérateur :

Depuis longtemps et en ce moment plus que jamais, j'ai assez de la littérature. Je voudrais débarrasser tous mes rayons des livres et n'y laisser que Claudel. Claudel est un monde, on peut y pénétrer sans crainte, il ne s'agit pas de prendre une attitude; il s'agit de vivre un certain temps une vie ardente et profonde.

$[\ldots]$

Pourtant je voudrais trouver un maître. [...] je voudrais trouver quelqu'un - écrivain ancien, femme, ou langage paysan - qui m'enseigne, qui m'aide à trouver la parole pour ce que je sens ${ }^{41}$.

Le symbolisme lui-même, devenu caduc, est à son tour dépassé :

Depuis la lecture (suffisante) des trois bouquins que tu sais ${ }^{42}$, il me semble que Verhaeren, Jammes et Laforgue sont partis dans des directions

39. Voir lettre de Jacques Rivière à Henri Fournier du 5 avril 1906, Correspondance I, p. 356-357 : «vas, tu la conquerras, $t a$ forme. »

40. Correspondance I, Henri Fournier à Jacques Rivière, 17 février 1906, p. 279.

41. Correspondance I, Henri Fournier à Jacques Rivière, 9 novembre 1906, p. 556-557.

42. Il s'agit de la correspondance de Laforgue, du Deuil des primevères de Jammes et des 
qui ne sont pas les miennes, et que (allons, tant mieux!) ils n'ont pas dit ce qu'il faut dire et que je veux dire.

D'où, d'abord, déception. Puis « tant mieux! ». Puis grande sympathie quand même et grande admiration surtout pour les premiers bouquins qui (me) les ont fait connaître et aimer ${ }^{43}$.

Au final il ne reste que la joie de se retrouver lancé dans la quête de l'écriture, de se sentir cheminer presque à l'aveugle sur une route encore vierge. Ce que Fournier cherchera désormais dans la lecture, ce ne sera plus, au fond, que la confirmation de sa solitude.

Il n'est pas si étonnant dès lors que ce lecteur acharné des poètes ait finalement développé l'art d'un conteur, lui qui devait lutter, alors qu'il amorçait la quête qui le mènerait au Grand Meaulnes, pour que le roman initialement projeté ne se transforme pas en vers ${ }^{44}$; il rêvait d'un roman « où les personnages ne sont que le flux et le reflux de la vie et de ses rencontres », ajoutant pour lui-même cette recommandation - tout de même extraite de ses Carnets à l'attention de son ami :

- Ne rien - même au fond - mépriser. S'y fondre, s'y confondre, s'y mêler. Y conformer sa pensée, y perdre sa pensée. Et la perdre ailleurs, le lendemain ${ }^{45}$.

Mais sa quête de l'écriture à travers la lecture avait déjà adopté cette forme longtemps cherchée : l'émotion que le symbolisme donnait, pure, évidente et nue, devait devenir l'objet toujours fuyant, instable, fugitivement aperçu, d'une perpétuelle recherche dont le ressac était le mouvement de l'existence même, aussi important, sinon plus, que l'objet poursuivi :

Ce qu'il y a de plus ancien, de presque oublié, d'inconnu à nous-mêmes : c'était cela que j'avais voulu faire de mon livre et c'était fou. C'était la folie du Symbolisme. Aujourd'hui cela tient dans mon livre la même place que dans ma vie : c'est une émotion défaillante, à un tournant de route, à un bout de paragraphe ${ }^{46}$.

Il faut enfin dire un mot du rôle joué par la correspondance comme échange et comme confrontation dans l'élaboration de cette conception de l'émotion littéraire comme surprise, dont Alain-Fournier donne l'exemple dans Le Grand Meaulnes, et que Rivière formule dans Le Roman d'aventure, qui lui fait pendant. Dès les premières années, on voit en effet s'exaspérer

Villes tentaculaires de Verhaeren.

43. Correspondance I, Henri Fournier à Jacques Rivière, 23 septembre 1905.

44. Voir lettre du 27 août 1905, Correspondance I, p. 111 : « Je ne te parle pas de mon roman à moi qui se transforme à mesure qu'il avance lentement, qui se transforme tellement qu'il devient des vers. "

45. Correspondance I, Henri Fournier à Jacques Rivière, 19 février 1906, p. 289.

46. Correspondance II, Henri Fournier à Jacques Rivière, 28 septembre 1910, p. 516. 
peu à peu la différence, au départ peut-être de nature ou de caractère, entre les deux épistoliers. L'un et l'autre se campent rapidement dans un rôle dont la partition s'écrit au fur et à mesure des mois de séparation : si la correspondance fait partie des choses lues et écrites à verser au bilan de l'expérience de vie, ce n'est pas en tant que lieu d'un épanchement ou d'un retour sur soi, mais comme l'espace d'une élaboration de soi dans la différenciation avec l'autre. Une lettre de Jacques Rivière du 13 janvier 1906 montre que ce processus fut très conscient. Il y propose à son ami «d'établir [leurs] comptes », en termes de pertes et profits tirés de leur entretien :

\footnotetext{
D'abord - un peu malgré toi - nous nous sommes formulés, en nous opposant l'un à l'autre. [...] l'essence d'une formule est d'être limitante, de ne pouvoir tout comprendre; par suite - si on la prétend rigoureuse - toute formule est nécessairement étroite et fausse. Mais son étroitesse et sa fausseté même sont un instrument de découverte. Car tout ce qui ne rentre pas dans la formule apparaît plus nettement dès qu'on pose cette formule, en s'opposant à elle. Tout ce qu'il y a de contradictoire en nous se révèle ainsi. Et cette révélation est de toutes la plus précieuse, s'il est vrai que notre vie est faite de l'oscillation entre les tendances contraires de notre moi profond ${ }^{47}$.
}

Et Rivière de rappeler ces formules : pour lui-même, « je suis quelqu'un qui sent le général ${ }^{48}$ »; pour Fournier : " "je suis quelqu'un qui sent la vie telle qu'elle se présente." J'ajoute : "et qui refuse d'intervenir pour organiser ses sensations". ${ }^{49} \gg$ Fournier reconnaît alors avoir participé à l'exercice mais, quant à travailler sur « sa » formule, comme son ami l'y encourage, il s'y refuse : «Ah non, par exemple... ! moi, monsieur, je ne fais pas de théorie; et je serai bien embarrassé de savoir où me classer. » Il ajoute :

C'est à moi que tu prétends me connaître, toi qui ne sais pas seulement combien de petites filles j'ai aimées, et la couleur de leurs robes, et de quels amours chaque fois différent, chaque fois unique, chaque fois nouveau sous le soleil ${ }^{50}$.

Ainsi, il joue son personnage; Rivière aussi, dont on a noté le besoin de netteté, de structure, comme garde-fou et point de départ à toute pensée du mouvant. Mais aussi, Fournier dénonce ici la correspondance comme incomplète et infidèle, non transparente. À une autre occasion, Rivière pourra s'en émouvoir à son tour : « Encore maintenant je sens avec douleur que je ne peux te suivre par où tu passes, que je ne vois que l'extérieur incohérent de ta transformation $^{51}$. » Mais l'habitude de lire et d'être lu - et parfois incompris - par l'autre

47. Ibid., p. 239.

48. Ibid.

49. Ibid., p. 241.

50. Correspondance I, Henri Fournier à Jacques Rivière, 22 janvier 1906, p. 250.

51. Correspondance II, Jacques Rivière à Henri Fournier, 29 mai 1909, p. 300. 
endurcit : elle enseigne à penser chaque vie comme un mystère, un ensemble opaque, pour laquelle chaque écrit est soit un coup de sonde donné à l'aveugle, soit une promenade dans un monde de signes complexes où rien ne se donne qu'au terme d'une longue fréquentation, et où rien de ce qui se donne n'est définitivement acquis. Lire l'autre, et dans les lettres de l'autre le soi étrange dont il vous renvoie le reflet, fait partie intégrante de cette double expérience existentielle et littéraire. Si l'aventure du lecteur consiste à chercher son chemin propre à travers les rencontres avec les auteurs d'une littérature vivante, en devenir, c'est aussi en revenir transformé vers l'autre, le correspondant élu qui vous renverra en pleine face votre nouvelle image, et vous forcera à chercher encore. Qu'il soit amical ou impitoyable, le regard de ce lecteur est le sel de l'existence, parce qu'il vous garde de la ratiocination narcissique et stérile, de l'enfermement dans le cercle de votre propre esprit; c'est pourquoi le jour où Fournier suggère à son ami de cesser toute correspondance, le temps pour lui d'entrer à l'École normale, la réaction de Rivière est violente :

Ta lettre me fait mal. Je n'y vois qu'une chose. Tu veux renoncer à cet entretien qui fait toute notre vie, toute ma vie, et cela au moment où j'en sens plus que jamais le besoin, où je suis tout éperdu et haletant après tes lettres, qui me soutiennent seules. [...]

Aujourd'hui, j'ai eu un grand coup au cœur. Sur le terrain de manœuvres, en me retournant, soudain, à quarante mètres, $j$ 'ai aperçu un potache : c'était toi, je t'ai reconnu tout de suite; il avait les mêmes gestes, le même chic. Je suis resté un moment, ne doutant pas que tu allais t'approcher. Puis, comme tu me regardais d'un air absent, je me suis avancé : il avait des lorgnons, et je crois qu'il était laid. Mais toute la journée, sur cette confusion, je me suis exalté et ravi, vivant de toi, avec toi, disant tant de choses et tant d'autres ${ }^{52}$.

Cette demi-hallucination de Rivière, apparaît comme une allégorie de sa relation avec Fournier telle qu'elle s'écrit dans leurs lettres; elle enseigne que son essence n'est pas dans la coïncidence ou la complicité : celui qui vous révèle et vous fait vivre, c'est celui qui vous arrache à vous-même et vous entraîne dans l'expérience toujours nouvelle des rencontres et des surprises, vous fait signe de loin puis vous présente un visage inconnu. Ainsi vivre, lire, être lu, sont autant d'aspects d'une même recherche, d'une même tension vers l'avenir; c'est en quoi la correspondance est le premier visage pris par l'aventure - l'aventure de deux hommes, de deux lecteurs, dont le célèbre essai de Rivière apparaîtra à la fois comme une métaphore et une projection en direction d'un devenir à jamais ouvert.

Julie Aucagne

Université de Bourgogne 